\title{
Levantamento e caracterização de espécies do nematoide das galhas em quivi no Rio Grande do Sul, Brasil
}

\author{
Lúcia Somavilla ${ }^{1}$, Cesar B. Gomes ${ }^{2}$, Jairo J. Carbonari³ \& Regina M. D. Gomes Carneiro ${ }^{4}$ \\ ${ }^{1}$ Universidade Federal de Pelotas, 96010-900, Capão do Leão, RS, Brasil; ${ }^{2}$ Laboratório de Fitopatologia, Embrapa Clima \\ Temperado, 96001-970, Pelotas, RS, Brasil; ${ }^{3}$ Ministério da Agricultura, Pecuária e Abastecimento, Serviço de Sanidade \\ Agropecuária, 90010-420, Porto Alegre, RS, Brasil; ${ }^{4}$ Embrapa Recursos Genéticos e Biotecnologia, 70849-000 Brasília, DF, \\ Brasil
}

Autor para correspondência: Lúcia Somavilla, e-mail: 1somavilla@hotmail.com

\begin{abstract}
RESUMO
Quarenta e quatro populações de Meloidogyne spp. provenientes de amostras de raízes de quivi, coletadas em pomares e viveiros do Estado do Rio Grande do Sul, Brasil, foram caracterizadas bioquimicamente utilizando-se a isoenzima esterase (Est). Meloidogyne arenaria Est A2 (Rm: 1,20, 1,28) foi a espécie mais frequente e ocorreu em 66,65\% das amostras. M. ethiopica com fenótipo Est E3 (Rm: $0,92,1,10,1,30)$ foi detectada em $16,66 \%$ das amostras, ocorrendo sempre em associação com outras espécies do gênero. Também foram identificadas M. javanica Est J3 (Rm: 1,00, 1,21, 1,35), M. hapla Est H1(Rm: 1,17), M. incognita Est I1 (Rm: 1,03) e Est I2 (Rm: 1,03, $1,10)$ em $29,9 \%, 16,66 \%, 3,33 \%$ e 9,79\% das amostras, respectivamente. Uma única população atípica de Meloidogyne sp. (3,33\%) com o fenótipo Est L3 (Rm: 1,00, 1,10, 1,30) foi detectada, porém nesse caso, não foi possível identificar a espécie com segurança, mesmo quando se recorreu a analise da configuração perineal das fêmeas.
\end{abstract}

Palavras-chave: Actinidia deliciosa, isoenzima esterase, nematoide das galhas.

\begin{abstract}
Survey and characterization of root-knot nematode species in kiwi in Rio Grande do Sul State, Brazil

Forty-four populations of Meloidogyne spp. obtained from a root-knot nematode survey on kiwi (Actinidia deliciosa) orchards and nurseries in Rio Grande do Sul State were characterized biochemically using esterase isoenzyme (Est). Meloiodgyne arenaria Est A2 (Rm: $1.20,1.28)$ was the most frequent species detected in this survey, occurring in $66.65 \%$ of the samples. Meloiodgyne ethiopica, with the phenotype E3 (Rm: 0.92, 1.10, 1.30) was detected in 16.66\% of the samples in association with other Meloidogyne species. Other species found were M. javanica Est J3 (Rm: 1.00, 1.21, 1.35), M. hapla Est H1 (Rm: 1.17), M. incognita I1 (Rm: 1.03) and I2 (Rm: 1.03, 1.10) identified in $29.9 \%, 16.66 \%, 3.33 \%$ and $9.79 \%$ of the samples, respectively. Only one atypical population presenting the phenotype L3 (Rm: $1.00,1.10,1.30)$ occurred in one orchard (3.33\%) but its identification was not possible even through the examination of the perineal patterns of females.
\end{abstract}

Key words: Actinidia deliciosa, isoenzyme esterase, root-knot nematode.

\section{INTRODUÇÃO}

Actinidia deliciosa (Chevalier) Liang \& Ferguson é uma espécie de fruteira popularmente conhecida como quivi e adaptada a climas temperados. Originária do sudeste da Ásia foi introduzida no Brasil no início dos anos 1970, porém somente em meados da década de 1980 adquiriu importância comercial no estado do Rio Grande do Sul. Em todo o Estado a cultura do quivizeiro apresenta uma área cultivada de 269,65 hectares e uma produção de 3.155,60 mil toneladas (EMATER, 2004). No entanto, não há dados atualizados sobre a área plantada hoje no Estado.

A cultura está sujeita a várias doenças, causadas principalmente por bactérias, fungos e nematoides. Entre os nematoides parasitas do quivi, destacam-se os nematoides das galhas (Meloidogyne spp.) sendo M. arenaria (Neal,
1989) Chitwood, 1949, M. hapla Chitwood, 1949, M. incognita (Kofoid \& White, 1919) Chitwood, 1949 e M. javanica (Treub, 1885) Chitwood 1949 as espécies mais frequentemente associadas à cultura (Vovlas \& Roca, 1976; Haygood et al., 1990; Pinochet, 1995; Philippi et al., 1995; Carneiro et al., 2007). Entretanto, alem dessas, uma outra espécie, M. ethiopica Whitehead, 1968 foi associada ao declínio da videira no Chile (Carneiro et al., 2007). Apesar da patogenicidade desta espécie no quivi, os danos parecem ser mais pronunciados em plantas de videira infectadas. De acordo com Magunacelaya (2005), a alta agressividade de M. ethiopica em videira tem resultado na morte das plantas ou em baixa produtividade dos pomares afetados.

Conforme as informações do Serviço de Sanidade Vegetal, do Ministério de Agricultura, Pecuária e Abastecimento do Rio Grande do Sul, na década de 1980, 
mudas de quivi importadas do Chile, foram introduzidas em vários viveiros da região serrana desse Estado. Dez anos após a introdução dessa cultura, Carneiro et al. (2003) verificaram nessa mesma região, a presença de plantas de quivi com redução no crescimento, no tamanho dos frutos e na produção, sistemas radiculares mal desenvolvidos, deformados e com muitas galhas. Os autores associaram esses sintomas a altas populações de uma espécie atípica de Meloidogyne spp. com fenótipo Est Ki3. Posteriormente, essas populações foram comparadas morfológica e bioquimicamente com uma população de $M$. ethiopica, proveniente do Quênia e outra população atípica (Est E3) proveniente do Chile. Devido às similaridades encontradas entre elas, as três foram identificadas como $M$. ethiopica (Carneiro et al., 2004).

Em levantamento realizado na cultura do quivi no Chile, entre 1992 e 1993, foram detectadas as espécies: M. hapla, M. incognita, M. arenaria e o fenótipo de esterase Ki3, típico de M. ethiopica (Philippi et al., 1996). Posteriormente, Carneiro et al. (2007) caracterizando populações de Meloidogyne provenientes de quivi e videira, desse mesmo país, identificaram M. ethiopica em $80 \%$ das amostras, detectando-se essa espécie desde o vale do Copiapó ao norte até Talca ao sul do Chile. Considerando a importância que $M$. ethiopica representa ao quivi e a videira e a falta de informações consistentes sobre sua distribuição e ocorrência no Brasil, objetivou-se neste trabalho, caracterizar, bioquímica e morfologicamente, as populações do nematoide das galhas, obtidas durante levantamento de campo conduzido em pomares e viveiros de quivi no estado do Rio Grande do Sul.

\section{MATERIAL E MÉTODOS}

Trinta amostras de raízes de quivi foram coletadas em 11 viveiros e 19 pomares comerciais, de 16 municípios do estado do Rio Grande do Sul, sendo os locais de coletas georreferenciados por GPS (Global Position System). A coleta das amostras foi realizada em parceria com o Serviço de Sanidade Vegetal do Ministério da Agricultura Pecuária e Abastecimento (MAPA). Os pomares amostrados foram indicados por este órgão do governo, por serem provenientes de mudas importadas do Chile na década de 1980, além disso, todos os viveiros que apresentavam registro no MAPA foram amostrados. As amostras obtidas foram avaliadas quanto à presença e identificação da(s) espécie(s) de nematoides das galhas (Meloidogyne spp).

As amostras foram coletadas percorrendo-se o terreno de cada pomar em zigue-zague, definindo um número mínimo de 10 pontos de coleta por hectare, onde a área dos pomares amostrados variou de 0,1 a 1 ha. As coletas foram realizadas na projeção da copa das plantas, a uma profundidade de até $25 \mathrm{~cm}$ da superfície, de onde foram retiradas 20 a $50 \mathrm{~g}$ de raízes novas de quivi com solo. Amostras de áreas com plantas exibindo sintomas de amarelecimento e/ou raquitismo, em reboleiras, foram coletadas separadamente (Tihohod, 2000). Nos viveiros, amostras compostas de 10 plantas foram obtidas a cada lote de 1000 mudas de quivi (Tihohod, 2000). Logo após a coleta, as amostras foram acondicionadas em sacos plásticos etiquetados e levadas ao Laboratório de Nematologia da Embrapa Clima Temperado - Pelotas RS, para análise e posterior caracterização da(s) espécie(s) de Meloidogyne eventualmente presentes.

A identificação das espécies foi baseada no fenótipo esterase (Est) utilizando-se a técnica descrita por Carneiro \& Almeida (2001). Quando as populações continham mais de uma espécie, as massas de ovos das respectivas fêmeas foram armazenadas, individualmente, em microtubos (ependorf) contendo solução salina $0,1 \%$, com o objetivo de purificar as espécies detectadas em mistura. As populações puras de Meloidogyne spp. foram inoculadas em plantas de tomateiro (Solanum lycopersicum L.) cv. Santa Cruz, mantidas em vasos com solo autoclavado, em casa de vegetação a $25 \pm 5^{\circ} \mathrm{C}$, para posteriores estudos bioquímicos e morfológicos.

Os fenótipos enzimáticos encontrados foram identificados por letras e números que corresponderam à letra inicial do nome específico de cada espécie de Meloidogyne, seguida do número de bandas (Esbenshade \& Triantaphyllou, 1990). A frequencia observada dos diferentes fenótipos foi expressa em percentagem. No caso isolado de uma população de Meloidogyne considerada atípica, referente a amostra do município de Nova Roma do Sul, não identificada com precisão por eletroforese, recorreu-se, adicionalmente, ao exame da configuração perineal de fêmeas adultas, como preconizado por Hartmann \& Sasser (1985).

\section{RESULTADOS}

O ponto de coleta mais setentrional foi em Erechim $\left(52^{\circ} 15^{\prime} 25^{\prime \prime} \mathrm{W}, 27^{\circ} 37^{\prime} 32^{\prime \prime} \mathrm{S}\right)$ e o extremo meridional foi em Caçapava do Sul $\left(53^{\circ} 26^{\prime} 38^{\prime \prime} \mathrm{W}, 30^{\circ} 57^{\prime} 21^{\prime \prime} \mathrm{S}\right)$. De Leste a Oeste, a amplitude foi de $52^{\circ} 18^{\prime} 11^{\prime \prime} \mathrm{W}$ $26^{\circ} 44^{\prime} 49^{\prime \prime}$ S Leste (Encruzilhada do Sul) a $54^{\circ} 30^{\prime} 28^{\prime \prime} \mathrm{W}$, $28^{\circ} 4^{\prime 2} 2$ 'S Oeste (Giruá). Detectou-se a presença do nematoide das galhas parasitando raízes de quivi em todas as amostras coletadas. Foram obtidas quarenta e quatro populações de Meloidogyne spp., as quais foram posteriormente caracterizadas bioquimicamente. Entre as populações estudadas, sete fenótipos esterásticos foram detectados nos diferentes locais (Tabela 1, Figura 1).

Dez bandas esterásticas foram encontradas nas diferentes populações de Meloidogyne spp. (Tabela 1, Figura 1). O fenótipo E3 (Rm: 0,92, 1,10,1,30), típico de $M$. ethiopica, foi detectado em $16,6 \%$ das amostras. O fenótipo A2 (Rm: $1,20,1,28)$ correspondente a $M$. arenaria, foi o mais frequente, sendo detectado em $66,65 \%$ das amostras. Os fenótipos J3 (Rm: 1,00, 1,21, 1,35) de M. javanica, H1 (Rm: 1,17) de M. hapla, I1 (Rm:1,03) e I2 (Rm: 
Levantamento e caracterização de espécies do nematoide das galhas ...

TABELA 1 - Fenótipos isoenzimáticos de esterase e suas respectivas percentagens de ocorrência observadas em 44 populações de Meloidogyne spp. provenientes de plantas de quivi coletadas em diferentes pomares do Rio Grande do Sul

\begin{tabular}{|c|c|c|c|c|}
\hline $\begin{array}{l}\text { Amostra } \\
\text { Pomares }\end{array}$ & Procedência (município) & Espécie & Fenótipo Esterase & Ocorrência(\%) \\
\hline \multicolumn{5}{|c|}{ POMARES } \\
\hline \multirow[t]{2}{*}{1} & Flores da Cunha & M. incognita & $\mathrm{I} 2$ & 88,3 \\
\hline & & M. ethiopica & E3 & 11,6 \\
\hline \multirow[t]{2}{*}{2} & Antônio Prado & M. arenaria & A2 & 90,7 \\
\hline & & M. javanica & $\mathrm{J} 3$ & 9,2 \\
\hline 3 & Antônio Prado & M. arenaria & A2 & 100,0 \\
\hline 4 & Cotiporã & M. arenaria & $\mathrm{A} 2$ & 100,0 \\
\hline 5 & Veranópolis & M. arenaria & $\mathrm{A} 2$ & 100,0 \\
\hline 6 & Erechim & M. arenaria & $\mathrm{A} 2$ & 100,0 \\
\hline 7 & Gaurama & M. arenaria & $\mathrm{A} 2$ & 100,0 \\
\hline 8 & Lagoa Vermelha & M. arenaria & $\mathrm{A} 2$ & 100,0 \\
\hline \multirow[t]{2}{*}{9} & Vacaria & M. hapla & $\mathrm{H} 1$ & 27,2 \\
\hline & & M. ethiopica & E3 & 72,7 \\
\hline \multirow[t]{2}{*}{10} & Vacaria & M. hapla & H1 & 90,0 \\
\hline & & M.javanica & $\mathrm{J} 3$ & 10,0 \\
\hline \multirow[t]{4}{*}{11} & São Marcos & M. arenaria & $\mathrm{A} 2$ & 32,4 \\
\hline & & M. incognita & $\mathrm{I} 1$ & 17,5 \\
\hline & & M. incognita & $\mathrm{I} 2$ & 47,1 \\
\hline & & M. javanica & $\mathrm{J} 3$ & 2,7 \\
\hline 12 & Caçapava do Sul & M. arenaria & $\mathrm{A} 2$ & 100,0 \\
\hline 13 & Santa Maria & M. arenaria & $\mathrm{A} 2$ & 100,0 \\
\hline \multirow[t]{2}{*}{14} & Encruzilhada do Sul & M. hapla & H1 & 45,9 \\
\hline & & M. ethiopica & E3 & 54,0 \\
\hline 15 & Giruá & M. arenaria & $\mathrm{A} 2$ & 100,0 \\
\hline \multirow[t]{2}{*}{16} & Farroupilha & M. arenaria & $\mathrm{A} 2$ & 11,7 \\
\hline & & M. javanica & $\mathrm{J} 3$ & 88,2 \\
\hline 17 & Farroupilha & M. javanica & $\mathrm{J} 3$ & 100,0 \\
\hline \multirow[t]{2}{*}{18} & Farroupilha & M. javanica & $\mathrm{J} 3$ & 95,0 \\
\hline & & M. ethiopica & E3 & 5,0 \\
\hline 19 & Nova Roma do Sul & Meloidogyne sp & L3 & 100,0 \\
\hline \multicolumn{5}{|c|}{ VIVEIROS } \\
\hline 20 & Farroupilha & M. javanica & $\mathrm{J} 3$ & 100,0 \\
\hline 21 & Farroupilha & M. arenaria & $\mathrm{A} 2$ & 100,0 \\
\hline 22 & Farroupilha & M. hapla & $\mathrm{H} 1$ & 100,0 \\
\hline 23 & Alto Feliz & M. hapla & $\mathrm{H} 1$ & 100,0 \\
\hline 24 & Alto Feliz & M. arenaria & $\mathrm{A} 2$ & 100,0 \\
\hline 25 & Alto Feliz & M. arenaria & $\mathrm{A} 2$ & 100,0 \\
\hline 26 & Alto Feliz & M. arenaria & $\mathrm{A} 2$ & 100,0 \\
\hline \multirow[t]{2}{*}{27} & Alto Feliz & M. arenaria & $\mathrm{A} 2$ & 66,0 \\
\hline & & M. ethiopica & E3 & 34,0 \\
\hline 28 & Alto Feliz & M. arenaria & $\mathrm{A} 2$ & 100,0 \\
\hline \multirow[t]{3}{*}{29} & Farroupilha & M. ethiopica & E3 & 42,8 \\
\hline & & M. javanica & $\mathrm{J} 3$ & 7,1 \\
\hline & & M. hapla & $\mathrm{H} 1$ & 50,0 \\
\hline \multirow[t]{2}{*}{30} & Farroupilha & M. incognita & $\mathrm{I} 2$ & 16,8 \\
\hline & & M. javanica & $\mathrm{J} 3$ & 83,2 \\
\hline
\end{tabular}

$1,03,1,10)$ de $M$. incognita, e uma população atípica de Meloidogyne sp. (Rm: 1,00, 1,10, 1,30), foram identificados em $29,9 \%, 16,66 \%, 3,33 \% 9,79 \%$ e $3,33 \%$ das amostras, respectivamente.

Nos pomares, foram detectadas 12 populações de $M$. arenaria com o fenótipo de esterase A2, correspondendo a $63,15 \%$ das amostras analisadas (Tabela 1). Uma população de $M$. incognita Est I1 e duas de $M$. incognita Est I2, corresponderam a 5,26\% e $10,52 \%$ das amostras, respectivamente. Encontraram-se ainda três populações de $M$. hapla com o fenótipo $\mathrm{H} 1$, seis de $M$. javanica Est J3, quatro de $M$. ethiopica Est E3 e uma população 


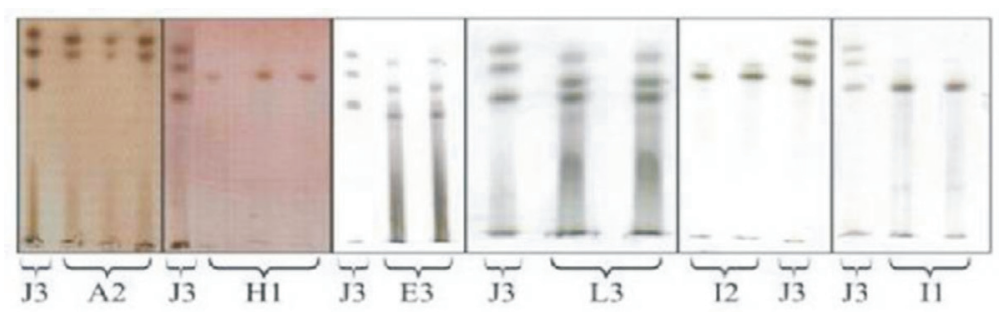

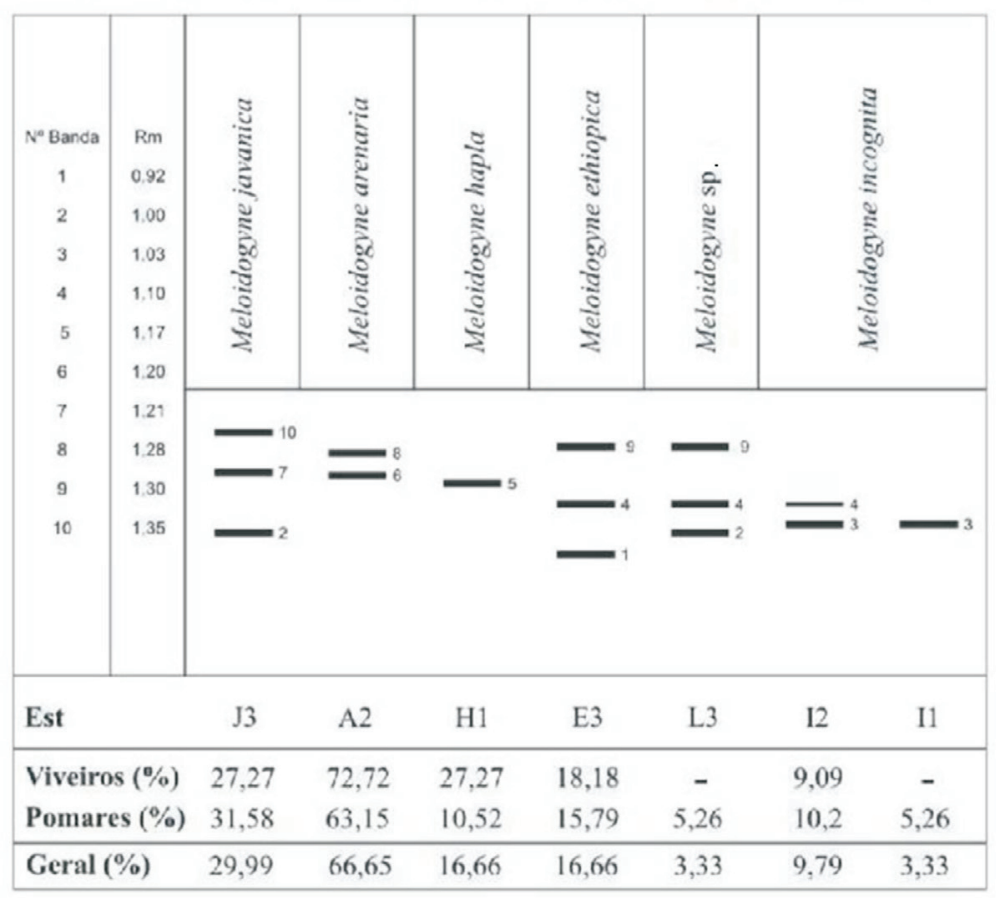

FIGURA 1 - Fenótipos de esterase (Est) e suas respectivas percentagens de ocorrência em 44 populações de Meloidogyne spp. coletadas em viveiros e pomares de quivi no Rio Grande do Sul. M. javanica (J3); M.arenaria (A2); $M$. hapla (H1); M. ethiopica (E3); Meloidogyne sp. (L3); $M$. incognita (I2) e $M$. incognita (I1) e $M$. javanica $(\mathrm{J} 3)$.
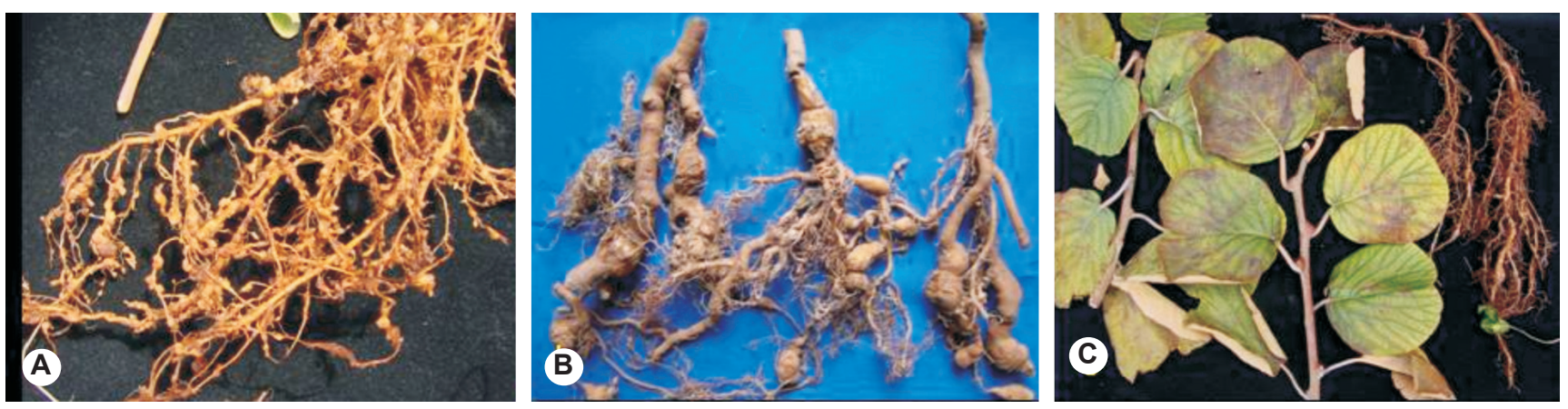

FIGURA 2 - A. Raízes de begônia, B. quivi com galhas causadas por Meloidogyne sp. (Est. L3); e C. folhas de quivi exibindo sintomas de necrose e escurecimento do limbo foliar, em plantas infectadas pela mesma população do nematoide.

atípica de Meloidogyne sp., correspondendo a 10,52\%, $31,58 \%, 15,79 \%$ e $5,26 \%$ das amostras analisadas, respectivamente. Nas onze amostras provenientes dos viveiros, foram identificadas seis populações de $M$. arenaria Est A2, três de $M$. javanica Est J3, três de $M$. hapla Est H1, uma de $M$. incognita com o fenótipo I2 e duas de $M$. ethiopica Est E3 que corresponderam, respectivamente, a $72,72 \%, 27,27 \%, 27,27 \%, 9,09 \%$ e $18,18 \%$ das amostras (Tabela 1 ).

\section{DISCUSSÃO}

Considerando-se a percentagem de ocorrência dos nematoides das galhas nas raízes de quivi, verificou-se a predominância de $M$. arenaria, tanto nos pomares como nos viveiros. No entanto, $M$. hapla, $M$. incognita, $M$. ethiopica são as espécies mais frequentes na cultura do quivi, em outros países (Watson et al., 1991; Philippi et al., 1995; Carneiro et al., 2007; Ma et al., 2007; Fengli \& Quiving, 
1990). Embora tenha sido observada maior ocorrência de M. arenaria no presente estudo, não foi possível associá-la com danos nas plantas de quivi amostradas.

O fenótipo E3, característico de M. ethiopica, foi encontrado em cinco municípios amostrados. Essa espécie, considerada agressiva a videira no Chile (Carneiro et al., 2007), teve sua ocorrência registrada no Brasil recentemente (Carneiro et al., 2003). Através das características morfológicas como a configuração perineal das fêmeas, esta espécie foi por muito tempo, confundida com M. incognita e M. arenaria. Bioquimicamente, o fenótipo esterástico E3 é específico de $M$. ethiopica e é usado para diferenciá-la de outras espécies do nematoide das galhas (Carneiro et al., 2004).

Foram identificadas seis populações de M. hapla com fenótipo Est H1, em quatro dos 16 municípios amostrados. Populações com este mesmo fenótipo já haviam sido identificadas no Brasil, em quivizeiro, nos estados de São Paulo e Rio Grande do Sul por Carneiro et al. (2000). Em vários países, essa espécie de nematoide das galhas é frequentemente relatada parasitando plantas de quivi. De acordo com Philippi et al. $(1995,1996)$ no Chile, as condições ambientais do Vale Central, com temperatura média anual de $14,9^{\circ} \mathrm{C}$ favorecem a ocorrência de $M$. hapla mas as plantas de quivi aparentemente toleram os danos causados pela espécie. Porém, na Nova Zelândia, Sale (1985) e Knight (2001), atribuíram o menor vigor e a baixa produtividade de quivi às populações de $M$. hapla presentes na cultura. Esta espécie também é considerada dominante nas áreas de cultivo de quivi na Coréia do Sul (Ma et al., 2007).

Uma população atípica de Meloidogyne sp. com três bandas esterásticas (Rm: 1,00, 1,10, 1,30), foi encontrada em quivi cv. Bruno, no município de Nova Roma do Sul (Figura 1). Plantas de begônia (Begonia sp.), coletadas sob a copa das mesmas plantas, também estavam parasitadas e exibiam o mesmo padrão de esterase L3. Por ocasião da amostragem, verificou-se que as plantas de quivi coletadas neste local, apresentavam amarelecimento das folhas seguido de necroses e seca progressiva dos bordos para o centro das folhas (Figura 2). Na região perineal das fêmeas desta população, observou-se a presença de estrias grossas, lisas e onduladas em ziguezague e um arco dorsal trapezoidal, semelhante a $M$. incognita, sem a presença do campo lateral. Entretanto não foi possível identificar essa espécie de Meloidogyne utilizando-se apenas essa característica morfológica. Esse mesmo fenótipo já foi relatado em quivi e em videira, no Chile por Philippi et al. (1998) e Carneiro et al. (2007). Foi detectado também em plantas de lavanda (Lavandula spica L.) no Rio Grande do Sul (Carneiro et al., 2000). Esbenshade \& Triantaphyllou (1985) detectaram o perfil L3 (=M3) em três populações provenientes da América do Sul e uma da Turquia. Infelizmente, a espécie ainda não foi devidamente identificada e, portanto, mais estudos devem ser realizados visando a esclarecer tal questão.
Tanto nas amostras coletadas nos pomares como nos viveiros, detectou-se a ocorrência de populações mistas de Meloidogyne spp., representando 33,3\% das amostras analisadas. Em todas as amostras em que $M$. ethiopica foi detectada, ocorreram também outras espécies como M. hapla, M. javanica, M. arenaria e $M$. incognita, demonstrando que a cultura do quivi é parasitada pelas espécies de Meloidogyne que ocorrem com maior freqüência em espécies frutíferas (El-Borai \& Duncan, 2005, Wehunt, 1984; El-Borai \& Duncan, 2005). Em levantamento recente realizado no Chile, Carneiro et al. (2007) também observaram a ocorrência de populações mistas de Meloidogyne na cultura do quivi e também da videira. Sabe-se que no Chile, a fiscalização e a interdição da produção de mudas infectadas, aparentemente não foram efetivas, reforça-se a hipótese de que $M$. ethiopica tenha sido introduzida no estado do Rio Grande do Sul em 1989, por mudas contaminadas importadas do Chile (Carneiro et al., 2003, 2007).

Embora a distribuição de $M$. ethiopica em quivi seja relativamente pequena na região estudada, essa espécie foi encontrada em pelo menos dois viveiros da Serra Gaúcha. Dado a proximidade das áreas de cultivo de quivi com as de videira, a última encontra-se sob iminente perigo devido a sua alta suscetibilidade ao nematoide. Em vista dos resultados relatados neste estudo referentes ao Rio Grande do Sul e considerando a importância dessa espécie de nematoide à produção vinícola brasileira, medidas sanitárias devem ser tomadas para impedir a sua disseminação, exigindo-se para tanto, rigor absoluto no plantio de mudas isentas de nematoides.

\section{AGRADECIMENTOS}

A primeira autora agradece à Coordenação de Aperfeiçoamento de Pessoal de Nível Superior - CAPES pela concessão da bolsa de estudo e ao Conselho Nacional de Desenvolvimento Científico e Tecnológico - CNPq pelo recurso concedido para a execução deste trabalho.

\section{REFERÊNCIAS BIBLIOGRÁFICAS}

Carneiro RMDG, Almeida MRA (2001) Técnica de eletroforese usada no estudo de enzimas dos nematoides das galhas para identificação de espécies. Nematologia Brasileira 25:35-44.

Carneiro RMDG, Almeida MRA, Guedes AC (2004) Detecção de Meloidogyne ethiopica e M. javanica em yacon no Distrito Federal. Fitopatologia Brasileira 29 (Supl.):82.

Carneiro RMDG, Almeida MRA, Cofcewicz ET, Magunacelaya JC, Aballay E (2007) Meloidogyne ethiopica, a major root-knot nematode parasitising Vitis vinifera and other crops in Chile. Nematology 9:635-641.

Carneiro RMDG, Almeida MRA, Quénéhervé P (2000) Enzyme phenotypes of Meloidogyne spp. populations. Nematology 2:645654. 
Carneiro RMDG, Gomes CB, Almeida MR, Gomes ACC, Martins I (2003) Primeiro registro de Meloidogyne ethiopica Whitehed, 1968 em plantas quivi no Brasil e reação em diferentes plantas hospedeiras. Nematologia Brasileira 27:152-158.

El-Borai FE, Duncan LW (2005) Nematode parasites of subtropical and tropical fruit ter crops. In: Luc M, Sikora RA, Bridge J (Eds.) Plant parasitic nematodes in subtropical and tropical agriculture. Wallingford UK. CAB International. pp. 467-492.

EMATER-RS (2004) Levantamento da fruticultura comercial do Rio Grande do Sul-2003/2004. Porto Alegre RS. Emater RS - ASCAR.

Esbenshade PR, Triantaphyllou AC (1985) Use of enzyme phenotypes for the identification of Meloidogyne species. Journal of Nematology 17:6-20.

Esbenshade PR, Triantaphyllou AC (1990) Isozyme phenotypes for the identifications of Meloidogyne species. Journal of Nematology 22:10-15.

Hartmann KM, Sasser JN (1985) Identification of Meloidogyne species on the basis of differential host test and perineal patterns morphology. In: Barker KR, Carter CC, Sasser JN (Eds.) An advanced treatise on Meloidogyne, Vol. 2. Methodology. North Carolina State University Graphics. Raleigh NC. pp. 69-77.

Haygood RA, Saunders JA, Miller JA (1990) Widespread occurrence of Meloidogyne incognita on quivifruit in the Coastal areas of South Carolina. Plant Disease 74:81.

Knight, KWL (2001) Plant parasitic nematodes associated with six subtropical crops in New Zealand. New Zealand Journal of Crop and Horticultural Science 29:267-275.
Ma KC, Jo YS, Kim BH, Lim DG (2007) Seasonal occurrence and aspects of root-knot nematodes in major quivifruit cultivation areas of Korea. Acta Horticulturae (ISHS) 753:719-724.

Magunacelaya JC (2005) Meloidogyne ethiopica y el cultivo de la vid em Chile In: $25^{\circ}$ Congresso Brasileiro de Nematologia. Piracicaba SP.

Philippi I, Latore BA, Pérez GF, Castillo L (1996) Identificaión de los nematodos del nudo (Meloidogyne spp.) del kivi por análisis de isoenzimas, en Chile. Fitopatologia 31:96-101.

Philippi I, Torres R, Latorre KA, Castillo L (1995) Meloidogyne spp. y Phytophthora $s p$. asociadas a kiwi en la zona central de Chile. Fitopatologia 29:1.

Pinochet J (1995) La selecion de patrones de frutales subtropicales e de clima templado frente a nematodos. In: $19^{\circ}$ Congresso Internacional de Nematologia Tropical, Rio Quente. Resumos e Anais, pp. 331-340.

Sale PR (1985) Kiwifruit culture. Wellington, New Zealand. Government Printer.

Tihohod D (2000) Nematologia Agricola Aplicada. 2 ed. Jaboticabal, São Paulo SP.

Vovlas N, Roca F (1976) Meloidogyne hapla su Actinidia chinensis in Italia. Nematologia Mediterranea 4:115-116.

Watson RN, Wilson EA, Marsden RS (1991) Distribution of plant-parasitic nematodes in the rhizosphere of kiwifruit. Acta Horticulturae 297:537-543.

Wehunt EJ (1984) Nematode parasites of peach and other tree crops. In: Nickle W (Ed.) Plant and insect nematodes. New York NY. Marcel Dekker. pp.435-455.

TPP 199 - Recebido 19 Outubro 2010 - Aceito 15 Abril 2011

Editor de Seção: Rosângela D'Arc Lima 